\title{
Efficacy and safety of CT-guided percutaneous pulsed radiofrequency treatment of the Gasserian ganglion in patients with medically intractable idiopathic trigeminal neuralgia
}

This article was published in the following Dove Press journal:

Journal of Pain Research

\section{Meng Lan* \\ Jia Zipu* \\ Shen Ying \\ Ren Hao \\ Luo Fang}

Department of Anesthesiology and Pain Management, Beijing Tiantan Hospital, Capital Medical University, Beijing, PR China

*These authors contributed equally to this work
Correspondence: Luo Fang Department of Anesthesiology and Pain Management, Beijing Tiantan Hospital, Capital Medical University, No II 9 West Road, South 4th Ring Road, Fengtai District, Beijing 100050, PR China

$\mathrm{Tel}+86$ I3 $61 \quad 1326978$

Email luofangwt@yahoo.com
Background: There is a lack of prospective studies for the long-term results of percutaneous pulsed radiofrequency (PRF) of the Gasserian ganglion in the treatment of patients with medically refractory trigeminal neuralgia (TN).

Methods and results: We prospectively observed the outcomes of 28 idiopathic TN patients (between July 2013 and July 2016) who received CT-guided percutaneous PRF treatment of the Gasserian ganglion. All of the patients had stopped responding to drug therapy before PRF treatment. The effective treatment standard was a reduction in the pain numeric rating scale (NRS) by $\geq 50 \%$ after the procedure. The postoperative NRS score decreased gradually from preoperative $7.6 \pm 0.8$ months to $1.5 \pm 2.4,0.2 \pm 0.4,0.2 \pm 0.4,0.1 \pm 0.4$, and $0.1 \pm 0.4$ at 1,3 , and 6 months and 1 and 2 years after the PRF treatment. The response rates at 1, 3, and 6 months were $85.7 \%$, and the rates at 12 months and 2 years were maintained at $78.6 \%$. No serious side effects were observed.

Conclusion: CT-guided PRF invention is an effective and safe technique for medically intractable idiopathic TN patients. This minimally invasive alternative treatment has the potential as a first-line therapy for TN.

Keywords: trigeminal neuralgia, pulsed radiofrequency, efficacy, safety

\section{Introduction}

Trigeminal neuralgia $(\mathrm{TN})$ is characterized by paroxysmal, sudden, unilateral, brief, electric shock-like, and recurrent pain in the facial region innervated by the trigeminal nerve. ${ }^{1}$ Refractory TN can affect the quality of life of patients and lead to depression.

Antiepileptic drugs, such as carbamazepine and oxcarbazepine, are utilized as first-line treatments for TN. However, in some patients, drug treatment is ineffective, and some patients cannot tolerate the side effects of antiepileptic drugs. There are multiple options, such as nerve block with local anesthetic and steroid, percutaneous retrogasserian glycerol injection, radiofrequency thermocoagulation, gamma knife, percutaneous balloon compression (PBC), and microvascular decompression (MVD) for patients refractory to drugs..$^{2-5}$ Nerve block is a simple and safe percutaneous procedure for $\mathrm{TN}$ patients. However, in order to achieve a certain clinical effectiveness, multiple repeated treatments are often needed with puncture-associated risks 
and drug-associated side effects. ${ }^{6,7}$ Retrogasserian glycerol injection, radiofrequency thermocoagulation, and $\mathrm{PBC}$ as nerve-damaging techniques may inevitably cause facial numbness, masseter muscle weakness, and other discomforts. Gamma knife therapy has a slower onset and lower response rate, while MVD treatment is not suitable for the elderly and weak patients. ${ }^{2,4,5,8,9}$ Therefore, it is difficult for patients to choose between treatments.

Pulsed radiofrequency (PRF) is a novel minimally invasive and nondestructive procedure used to treat chronic pain disorders. The temperature during the procedure will not exceed $42^{\circ} \mathrm{C}$, so that it will not cause target tissue injury. ${ }^{10,11} \mathrm{Up}$ to now, there is a lack of prospective studies for the long-term results of percutaneous PRF of the Gasserian ganglion in the treatment of patients with medically refractory TN. We previously reported that percutaneous PRF treatment under CT guidance has some efficacy in refractory $\mathrm{TN}$ patients who do not respond to conservative therapy, including drugs and nerve block; we found that the response rates at 1,3 , and 6 months and 1 year were $69 \%{ }^{12}$ Chua et al performed a retrospective study and found that $73.5 \%$ of patients obtained excellent or satisfactory pain relief ( $\geq 50 \%$ pain relief) 1 year after PRF. ${ }^{13}$ However, in TN patients who are drug-refractory, the effectiveness of PRF intervention has not yet been prospectively evaluated. Cruccu et al proposed three diagnostic categories including classical TN with demonstration of morphologic changes in the trigeminal nerve root from vascular compression, secondary $\mathrm{TN}$ with an identifiable underlying neurologic disease, while TN of unknown etiology is labeled idiopathic TN. ${ }^{14}$ We hypothesized that PRF intervention as a novel neuromodulation treatment may have a fairly satisfactory response rate in drug-refractory idiopathic $\mathrm{TN}$ patients and conducted the current prospective study.

\section{Methods}

The study protocol and informed consent forms were approved by the Medical Ethics Committee of Beijing Tiantan Hospital, which is affiliated with Capital Medical University (no kylw-2010-014). The study was registered in the Chinese Clinical Trial Registry (ChiCTR) (registration number: ChiCTR-ONRC-12002939). All the patients signed the informed consent form.

Inclusion criteria: 1) age $\geq 18$ years; 2) recurrent paroxysmal or continuous unilateral facial pain without demonstration on MRI of neurovascular compression and morphological changes in the trigeminal nerve root; ${ }^{14} 3$ ) preoperative pain NRS score $\geq 7 ; 4$ ) patients in whom oral carbamazepine did not control pain or who could not tolerate the side effects of the drugs; and 5) patients who could cooperate.

Exclusion criteria: 1) abnormal routine blood, blood biochemistry, chest film, or electrocardiogram results; 2) puncture site infection; 3) coagulopathy; 4) history of local anesthetic allergy; 5) pregnancy or lactation; 6) history of mental disorders; 7) history of narcotic drug abuse; and 8) previous nerve block, PRF, radiofrequency thermocoagulation, balloon compression, retrogasserian glycerol injection, gamma knife, peripheral trigeminal nerve neurotomy or neurolysis injection, or MVD.

\section{Trial procedure}

The patient was in the supine position on the CT scanner bed. Routine monitoring, including blood pressure, heart rate, electrocardiogram, and pulse oxygen saturation, was performed. The disposable negative plate of the radiofrequency generator (PMG-230; Baylis Medical Inc., Montreal, QC, Canada) was placed on the abdominal skin. Oxygen inhalation during the procedure was through a nasal tube.

All patients were sedated before puncture by intravenous propofol at a dose of $0.75 \mathrm{mg} / \mathrm{kg}$. Ondansetron was administered to prevent nausea and vomiting. The puncture point was $\sim 3 \mathrm{~cm}$ outside the corner of the mouth on the affected side. Local infiltration anesthesia was performed with $1 \%$ lidocaine after routine disinfection and draping. Using Hartel's forward approach, the puncture was performed toward the ipsilateral foramen ovale with the guidance of spiral CT (Somatom; Siemens Company, Munich, Germany) scanning ( $2 \mathrm{~mm}$ /layer) and 3-D reconstructed images (Figure 1) with a $10 \mathrm{~cm}$ trocar with a $5 \mathrm{~mm}$ noninsulated tip (PMF-21-100-5; Baylis Medical Inc.). When the trocar was accurately piercing the foramen ovale, the physician removed the stylet and inserted the radiofrequency electrode (PMK-21-100; Baylis Medical Inc.). Electrical stimulation of $0.1-0.2 \mathrm{~V}$ at $50 \mathrm{~Hz}$ was used to test the feeling threshold and $2 \mathrm{~Hz}$ of electrical stimulation was used to test the motor threshold. The depth and direction of the trocar were adjusted in relation to the trigeminal nerve region feelings and mandibular movements of patients to ensure accurate puncture.

The radiofrequency generator was set at manual pulse mode. The upper temperature limit was set at $42^{\circ} \mathrm{C}$. The assistant nurse gradually increased the output voltage to the highest voltage level that could be tolerated by the patient (no obvious pain) for 360 seconds. ${ }^{15,16}$ After 1 month, patients who did not respond to PRF treatment could choose other 

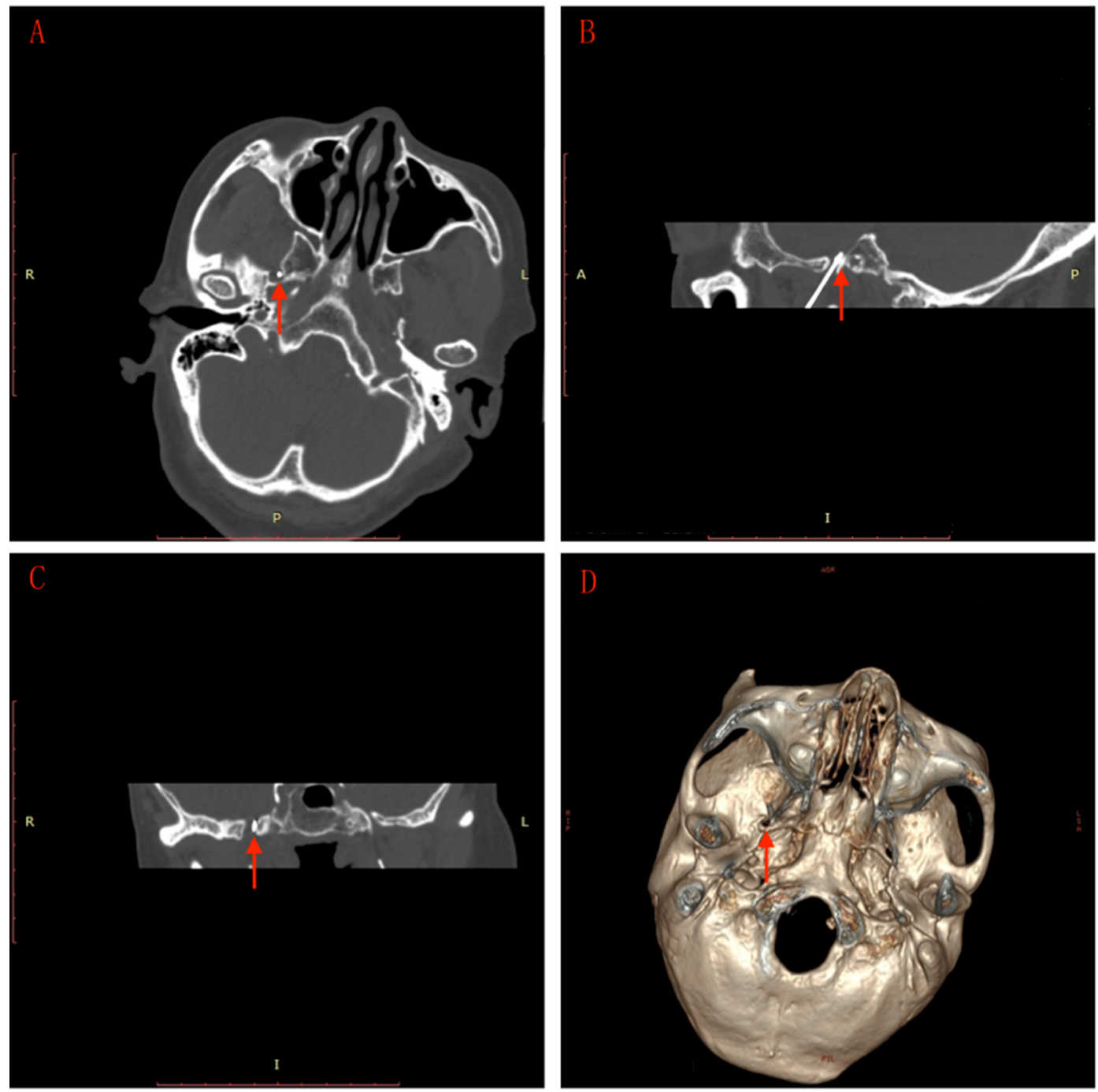

Figure I CT-guided foramen ovale puncture.

Notes: (A) Base of the skull shows the needle (arrow) entering the foramen ovale. (B) Sagittal view shows the needle (arrow) entering the foramen ovale. (C) Coronal view shows the needle (arrow) entering the foramen ovale. (D) 3-D reconstruction of the skull base shows the needle (arrow) entering the foramen ovale. Image scale: I unit $=\mathrm{Icm}$.

treatments, such as conventional radiofrequency thermocoagulation and MVD. If patients had initial effectiveness but experienced relapse later, they could choose PRF retreatment, radiofrequency thermocoagulation, or MVD.

\section{Data collection}

\section{Preoperative data}

Data regarding age, gender, disease duration, laterality, branch affected, comorbidities, pain NRS score (0: no pain at all; 10: the most severe pain imaginable), ${ }^{17}$ and dose of carbamazepine were recorded.

\section{Intraoperative data}

Data regarding operative duration, sensory and motor stimulation voltage, output voltage during treatment, tissue resistance immediately before and after PRF treatment, and complications, such as hematoma, were recorded.

\section{Postoperative data}

At 1, 7, and 14 days; 1,3 , and 6 months; and 1 and 2 years after the operation, the pain NRS score, carbamazepine dose, side effects, and complications were determined by follow-up telephone calls by a trained neurologist. The time to initial effectiveness of PRF treatment and time of carbamazepine treatment cessation were recorded. At months 1, 3, 6, and 12 and year 2, the efficacy rates were calculated (the number of patients who responded to PRF treatment/the total number of patients*100\%).

The effective treatment standard was a reduction in the pain NRS score of $\geq 50 \%$ after the PRF procedure. ${ }^{18}$ The 
recurrence criterion was defined as an NRS score returning to $\geq 50 \%$ of the preoperative NRS score.

Remedial treatment of patients who did not exhibit treatment efficacy 1 month after PRF treatment and retreatment of patients who suffered recurrent pain were also recorded.

\section{Statistical analyses}

Statistical analyses were performed using SPSS 20.0 (SPSS Inc., Chicago, IL, USA). Normally distributed data are expressed as the mean \pm standard deviation $(\mathrm{x} \pm \mathrm{SD})$ and were compared using single-factor analysis of variance. Non-normally distributed data are expressed as the median (interquartile range) and differences were compared with the Mann-Whitney $U$ test. A value of $P<0.05$ indicated a statistically significant difference.

\section{Results}

\section{Patient demographics}

Between July 2013 and July 2016, 42 consecutive idiopathic TN patients treated in the Department of Pain at Beijing Tiantan Hospital were screened. Twenty-eight eligible patients were included in this study. Fourteen patients were excluded since eight patients met exclusion criterion and six patients declined to participate. Nine patients were males and 19 were females. Their mean \pm SD age was $57 \pm 16$ years at the time of PRF treatment. Before treatment, the mean \pm SD NRS score was $7.6 \pm 0.8$. The median (interquartile range) dosage of carbamazepine was 600 (300-900) mg/day. The patient characteristics are presented in Table 1.

\section{Intraoperative data and treatment effect}

The operative duration was $32 \pm 9$ minutes. The $50 \mathrm{~Hz}$ sensory stimulation voltage and $2 \mathrm{~Hz}$ motor stimulation voltage were both $0.1(0.1-0.1) \mathrm{V}$ and the output voltage during the treatment was $52 \pm 18 \mathrm{~V}$. The tissue resistance just before PRF treatment was $284 \pm 29 \Omega$, while it was $283 \pm 29 \Omega$ immediately after the treatment.

All 28 TN patients successfully underwent foramen ovale puncture under the guidance of CT as well as PRF treatment. All 28 patients were followed in this study for 2 years. The flow chart and outcomes of the study are shown in Figure 2.

The postoperative NRS score decreased gradually after PRF treatment (Figure 3 and Table 2). Twenty-four (85.7\%) TN patients obtained effective pain relief after the PRF treatment compared with preoperative NRS scores at months 1 , 3 , and 6 postoperatively (the response rate was $85.7 \%$ up to 6 months after the treatment). Two ( $8 \%$ ) of these 24 patients
Table I The demographics of patients treated with PRF intervention

\begin{tabular}{|l|l|}
\hline Patients & $(\mathbf{n = 2 8})$ \\
\hline Age (years, $x \pm$ SD) & $57 \pm 16$ \\
Male/female (n) & $9 / 19$ \\
Disease duration (years, median & $3(1.375-4.375)$ \\
[interquartile range]) & \\
Left/right (\%), branch affected & $12 / 16$ \\
I (n) & $\mathrm{I}$ \\
II (n) & $\mathrm{I}$ \\
I+II (n) & $\mathrm{I}$ \\
I+II+III (n) & $\mathrm{I}$ \\
II+III (n) & $\mathrm{II}$ \\
III (n) & 13 \\
Preoperative carbamazepine dose & $600(300-900)$ \\
(mg/day, median [interquartile range]) & \\
Preoperative NRS comorbidities & $7.6 \pm 0.8$ \\
Hypertension (n) & 10 \\
Coronary disease (n) & 4 \\
Diabetes mellitus (n) & $\mathrm{I}$ \\
\hline
\end{tabular}

Abbreviations: NRS, numeric rating scale; PRF, pulsed radiofrequency.

suffered pain recurrence at 8 and 10 months, respectively. The response rate at 1 and 2 years was $78.6 \%$. The pain NRS scores of the two relapse patients significantly decreased after one patient underwent MVD and the other received PBC treatment. The median (interquartile range) time to the initial response after PRF treatment was 1 (0-30) day.

Four patients who exhibited treatment efficacy 1 month after PRF treatment reported that their pain intensity increased right after the PRF treatment and maintained a short-term increase for $\sim 8.5(6-10.75)$ days. One patient in the ineffective treatment group suffered postoperative pain with an increased NRS score for 7 days. The dosage of carbamazepine in the abovementioned five patients had to be increased during the period of increased pain.

Postoperative use of carbamazepine decreased gradually after the percutaneous PRF treatment during the follow-ups (Figure 4 and Table 2). Among the 24 patients who exhibited treatment efficacy, 16 patients gradually decreased their use of carbamazepine and eventually (at NRS $=0$ ) stopped using carbamazepine at 22 (14-30) days during the follow-up period. NRS score decreases of $\geq 50 \%$ were found in the other eight patients and their carbamazepine doses were also gradually reduced to low doses (100-300 mg/day) for pain control.

Four patients (14.3\%) failed to respond to percutaneous PRF treatment at month 1 postoperatively. All four patients who demonstrated treatment ineffectiveness selected neurodestructive radiofrequency thermocoagulation treatment. After 


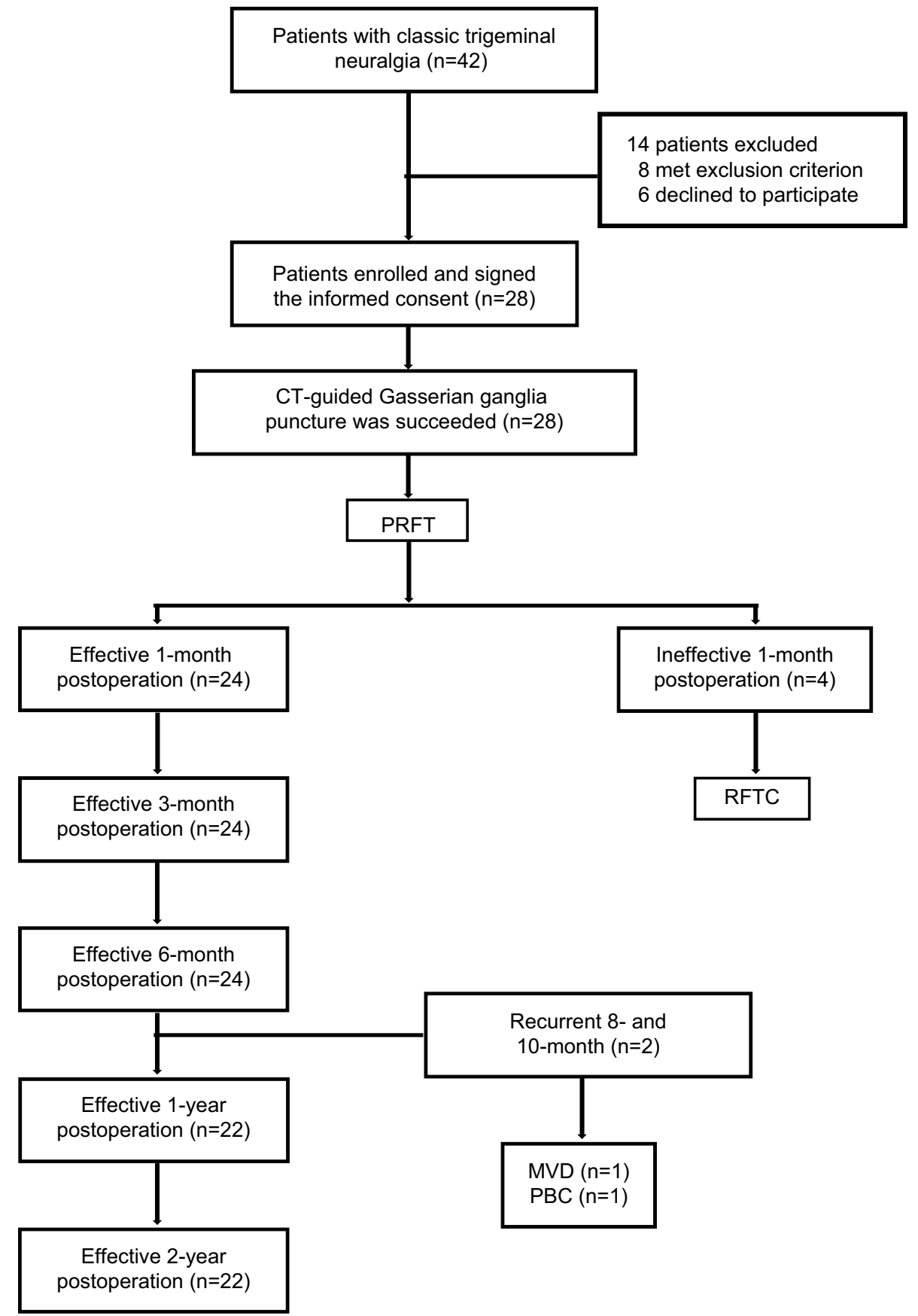

Figure 2 The flow chart and outcomes of the study.

Abbreviations: MVD, microvascular decompression; PBC, percutanous balloon compression; PRFT, pulsed radiofrequency treatment; RFTC, radiofrequency thermocoagulation. .

the treatment, the mean NRS score decreased to 0 and patients gradually stopped using carbamazepine after the procedure.

\section{Side effects and complications}

Two patients suffered from mild postoperative dizziness, nausea, and vomiting, and two patients felt only mild dizziness after the treatment. Those side effects spontaneously remitted in 1-2 hours and did not require special processing. One patient suffered from facial varicella zoster virus infection on the affected side 3 days after treatment, which had a 10-day course. The four patients who received radiofrequency thermocoagulation treat- 


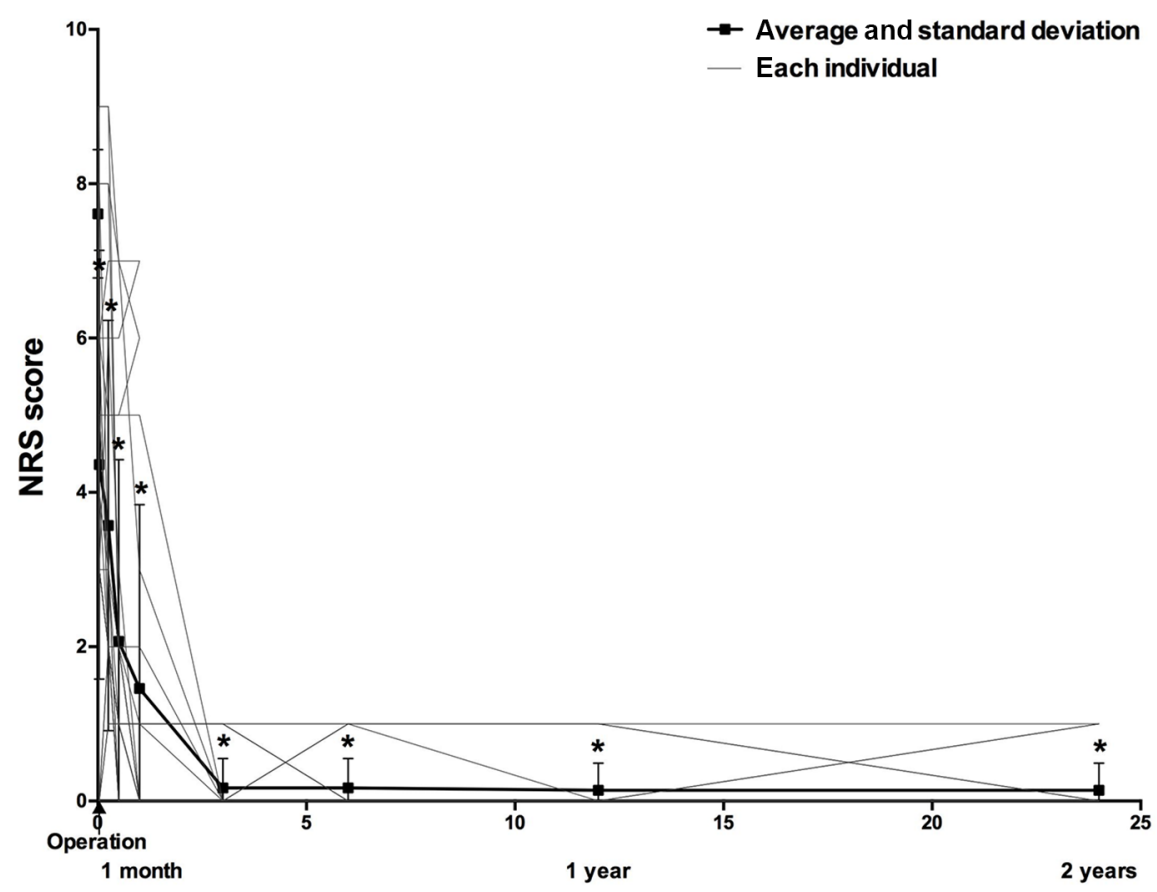

Figure 3 Changes in NRS score after PRF treatment.

Notes: I unit of length $=I$ month $=30$ days. ${ }^{*}$ Compared with preoperative NRS, $P<0.01$.

Abbreviations: PRF, pulsed radiofrequency, NRS, numeric rating scale.

ment suffered serious facial dysesthesia and masseter weakness.

\section{Discussion}

Encouragingly, this study showed that most $(85.7 \%)$ of the idiopathic $\mathrm{TN}$ patients who were unresponsive to prior medicine therapy obtained effective pain relief, which was defined as a postoperative NRS score $=0$ or a decrease in the NRS score by more than 50\% 6 months after the PRF intervention. Up to 2 years after PRF, the response rate was maintained at 78.6\%. Since 2003, Van Zundert et al first proposed that PRF technique can reduce the pain in five TN patients ${ }^{19}$ and a few scholars have conducted relevant studies. ${ }^{11-13}$ The response rate in this study was slightly higher than we previously reported (69\%) 1 year after PRF treatment in refractory TN patients who were unresponsive to drugs and nerve block. ${ }^{12}$ Similar to a retrospective study, Chua et al also found that PRF treatment was effective for TN patients. ${ }^{13}$ However, PRF has not been recommended as a therapeutic method in TN treatment guidelines due to lack of high level of evidence recommendation from clinical studies. Only two of 24 effective patients $(8 \%)$ experienced recurrence in this study at 8 or 10 months after the procedure; a curative effect was maintained in the other $22(92 \%)$ patients during the 2-year follow-up period. This result suggests that PRF intervention was fairly effective.

A series of large-scale prospective studies for the long-term results of conventional percutaneous destructive radiofrequency thermocoagulation in the treatment of TN patients have been reported with an acute success rate of $97.6 \%-99 \%{ }^{20-24}$ Sharma et al performed a systematic review and showed that the initial success rate with MVD for medically refractory TN was $96 \%(95 \%$ CI $93.3 \%-98.6 \%){ }^{25}$ Obviously, radiofrequency thermocoagulation and MVD resulted in superior rates of pain relief when compared to the PRF treatment reported in this study. However, PRF as the nondestructive and more minimally invasive technique will be more easily accepted by patients than destructive treatment and more invasive open surgery. Whether PRF treatment can become an alternative treatment technique for TN patients deserves further evaluation.

Nerve block treatment for TN always requires multiple repeated treatments to achieve a certain clinical efficacy, ${ }^{6,7}$ and traditional nerve block treatment has the disadvantages 


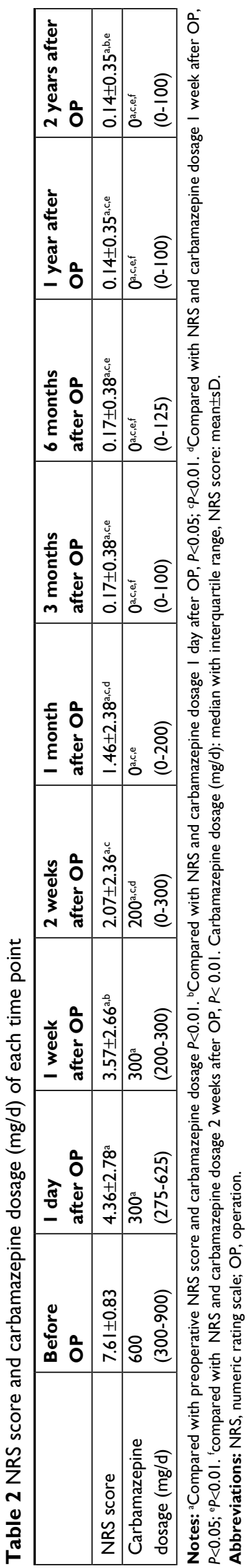

of puncture risks and drug-associated side effects. Additionally, multiple nerve block treatments can create problems with increased costs and radiation exposure. In this study, the response rate of one-time PRF treatment at 1 year reached up to $78.6 \%$, a very different efficacy was reported that the response rate of one-time nerve block treatment for TN patients is low (25\%), and $75 \%$ of the patients had to receive repeated blockade as the pain increased after the first blockade during one year of follow-up. ${ }^{7}$ By contrast, the pain relief efficacy of a single PRF treatment for TN in this study is promising, and it has the potential as a first-line therapy for $\mathrm{TN}$.

Consistent with previous studies, ${ }^{26,27}$ the pain intensity in TN patients gradually decreased rather than disappeared completely. We speculate that the neuromodulation action of the PRF electric field was gradually effective and that, as a result, pain relief was achieved over time. Furthermore, five patients reported increased pain intensity immediately after PRF treatment. The short-term pain increase may have been due to puncture injury or stimulation by high-voltage electric fields at the target location. Further investigation is warranted to determine whether at the end of the treatment, just before withdrawing the trocar, slow injection of steroids and local anesthetics can reduce the postoperative pain increase..$^{28,29}$

One of the important steps of the PRF procedure is puncture of the foramen ovale. With the assistance of CT technology, the success rate of puncture was $100 \%$ in this study. The mean operative time in this study was short, largely because the procedure was under the guidance of spiral CT three-dimensional reconstruction technology, which simplified the puncture process.

During the foramen ovale puncture, patients needed local anesthesia and sedation. The PRF treatment itself was painless and could be performed without anesthesia. Even for aged, frail patients with multiple medical comorbidities, this therapeutic modality is well tolerated. None of the patients in the study required hospitalization. However, a brief observation after the operation was necessary before patient departure from the hospital since four in $28(14 \%)$ patients suffered dizziness, nausea, and vomiting after the surgery. Fortunately, those side effects were mild and resolved rapidly. Consistent with previous studies, ${ }^{12,13,26,27} \mathrm{CT}$-guided PRF is a safe procedure with no serious intraoperative or postoperative side effects or complications detected.

The exact pain relief mechanisms of PRF treatment in TN remain unclear. In-depth experimental studies to investigate the effects and mechanism of PRF treatment in the TN model are needed. Whether this procedure alleviated the mechanical 


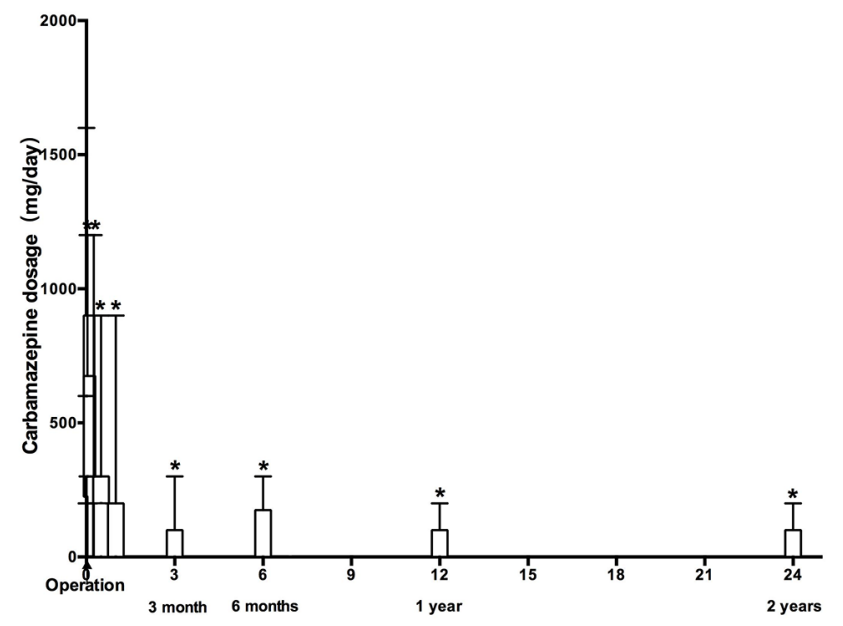

Figure 4 Changes in dose of carbamazepine after PRF treatment.

Notes: $I$ unit of length $=I$ month $=30$ days. ${ }^{*}$ Compared with preoperative dose of carbamazepine, $P<0.0$ I.

Abbreviation: PRF, pulsed radiofrequency.

compression of the trigeminal nerve by vascular structures was not known because we merely included idiopathic TN without demonstration of morphologic changes in the trigeminal nerve root from vascular compression and no patients underwent any MRIs after the PRF treatment in this study.

This study has several limitations. We merely included 28 patients and examined the efficacy of PRF for only two years after treatment. Larger amount cases and longer follow-ups would provide more significant results with regard to the effectiveness of PRF treatment in TN. This singlecenter prospective cohort study revealed that PRF invention is effective in idiopathic $\mathrm{TN}$ who had not responded to medication; however, the evidence base is weak. More high-quality clinical trials, such as multicenter, randomized, controlled studies, are still needed to evaluate the effectiveness and safety of the PRF technique. For example, further researches comparing the efficacy of repeated nerve block or other contemporary options (such as MVD) and PRF in TN patients refractory to drugs are needed. The median duration of idiopathic TN disease in this study was 3 (1.375-4.375) years. There is a very large range for disease duration. The correlation between length of disease duration and curative effect is worth further study. Furthermore, studies of a larger sample size are needed to compare the differences in patients who did respond to PRF treatment vs who did not respond. Pain is just a subjective experience; for example, quality of life by the Short Form-12 (SF-12) and distress by the Hospital Anxiety and Depression Scale should be evaluated in these patients. ${ }^{30}$ Other than subjective indicators, such as pain relief, objective evaluation methods, such as electrophysiological examination, warrant further study to obtain additional information with respect to changes in the trigeminal nerve. Other parameters, such as treatment duration of PRF, waveform, pulse width, frequency, and combined PRF and CRF treatment of the Gasserian ganglion at $60^{\circ} \mathrm{C}$ or $65^{\circ} \mathrm{C}$, which have been reported to be effective in idiopathic TN patients, ${ }^{15,31}$ should also be investigated in the future.

In conclusion, PRF treatment was an effective, safe, and nondestructive method for medically intractable TN patients. Early PRF treatment can be considered as a first-line choice before more invasive treatments, such as neurodestructive methods and MVD surgery. Whether PRF treatment is effective cannot currently be determined preoperatively. Age, gender, illness course, comorbidities, and other risk factors for ineffective treatment should be assessed in the future to aid pain physicians in developing individualized treatment plans for TN patients.

\section{Acknowledgments}

Category for which the manuscript is being submitted: Prospective cohort study.

This research was supported by Beijing Municipal Administration of Hospitals Clinical Medicine Development of Special Funding Support (No XMLX201707) and Highlevel Technical Personnel Training Program of the Beijing Municipal Health System (2011-3-034).

\section{Data sharing statement}

We intend to share all the collected individual de-identified participant data in the clinical trial. Other study-related documents including study protocol, informed consent, and case report form will be made available. The data will be accessible by writing to luofangwt@yahoo.com. The data will be made available 9 months after publication and will become unavailable 36 months after publication.

\section{Disclosure}

The authors report no conflicts of interest in this work.

\section{References}

1. Zakrzewska JM, Linskey ME. Trigeminal neuralgia. BMJ. 2014;348:g474.

2. Maarbjerg S, Di Stefano G, Bendtsen L, Cruccu G. Trigeminal neuralgia - diagnosis and treatment. Cephalalgia. 2017;37(7):648-657. 
3. Henson CF, Goldman HW, Rosenwasser RH, et al. Glycerol rhizotomy versus gamma knife radiosurgery for the treatment of trigeminal neuralgia: an analysis of patients treated at one institution. Int J Radiat Oncol Biol Phys. 2005;63(1):82-90.

4. Wang DD, Raygor KP, Cage TA, et al. Prospective comparison of long-term pain relief rates after first-time microvascular decompression and stereotactic radiosurgery for trigeminal neuralgia. $J$ Neurosurg. 2018;128(1):68-77.

5. Koning MV, Koning NJ, Koning HM, van Kleef M. Relationship between sensory stimulation and side effects in percutaneous radiofrequency treatment of the trigeminal ganglion. Pain Pract. 2014;14(7):581-587.

6. Nader A, Kendall MC, De Oliveria GS, et al. Ultrasound-guided trigeminal nerve block via the pterygopalatine fossa: an effective treatment for trigeminal neuralgia and atypical facial pain. Pain Physician 2013;16(5):E537-545.

7. Elsheikh NA, Amr YM. Calcitonin as an additive to local anesthetic and steroid injection using a modified coronoid approach in trigeminal neuralgia. Pain Physician. 2016;19(7):457-464.

8. Kotecha R, Kotecha R, Modugula S, et al. Trigeminal neuralgia treated with stereotactic radiosurgery: The effect of dose escalation on pain control and treatment outcomes. Int J Radiat Oncol Biol Phys. 2016;96(1):142-148

9. Ko AL, Ozpinar A, Lee A, Raslan AM, McCartney S, Burchiel KJ. Long-term efficacy and safety of internal neurolysis for trigeminal neuralgia without neurovascular compression. J Neurosurg. 2015;122(5):1048-1057.

10. Snidvongs S, Mehta V. Pulsed radio frequency: a non-neurodestructive therapy in pain management. Curr Opin Support Palliat Care. 2010;4(2):107-110.

11. Erdine S, Ozyalcin NS, Cimen A, Celik M, Talu GK, Disci R. Comparison of pulsed radiofrequency with conventional radiofrequency in the treatment of idiopathic trigeminal neuralgia. Eur J Pain. 2007;11(3):309-313.

12. Fang L, Tao W, Jingjing L, Nan J. Comparison of high-voltage- with standard-voltage pulsed radiofrequency of Gasserian ganglion in the treatment of idiopathic trigeminal neuralgia. Pain Pract. 2015;15(7):595-603.

13. Chua NH, Halim W, Beems T, Vissers KC. Pulsed radiofrequency treatment for trigeminal neuralgia. Anesth Pain Med. 2012;1(4) 257-261.

14. Cruccu G, Finnerup NB, Jensen TS, et al. Trigeminal neuralgia: New classification and diagnostic grading for practice and research. Neurology. 2016;87(2):220-228.

15. Ali Eissa AA, Reyad RM, Saleh EG, El-Saman A. The efficacy and safety of combined pulsed and conventional radiofrequency treatment of refractory cases of idiopathic trigeminal neuralgia: a retrospective study. J Anesth. 2015;29(5):728-733.

16. Kim JH, Yu HY, Park SY, Lee SC, Kim YC. Pulsed and conventional radiofrequency treatment: which is effective for dental procedure-related symptomatic trigeminal neuralgia? Pain Med. 2013;14(3):430-435.

17. Lemos L, Alegria C, Oliveira J, Machado A, Oliveira P, Almeida A. Pharmacological versus microvascular decompression approaches for the treatment of trigeminal neuralgia: clinical outcomes and direct costs. J Pain Res. 2011;4:233-244.
18. Jin HS, Shin JY, Kim YC, et al. Predictive Factors Associated with Success and Failure for Radiofrequency Thermocoagulation in Patients with Trigeminal Neuralgia. Pain Physician. 2015;18(6):537-545.

19. Van Zundert J, Brabant S, Van de Kelft E, Vercruyssen A, Van Buyten JP. Pulsed radiofrequency treatment of the Gasserian ganglion in patients with idiopathic trigeminal neuralgia. Pain. 2003;104(3):449-452.

20. Taha JM, Tew JM Jr, Buncher CR. A prospective 15-year follow up of 154 consecutive patients with trigeminal neuralgia treated by percutaneous stereotactic radiofrequency thermal rhizotomy. $J$ Neurosurg. 1995;83(6):989-993.

21. Zakrzewska JM, Jassim S, Bulman JS. A prospective, longitudinal study on patients with trigeminal neuralgia who underwent radiofrequency thermocoagulation of the Gasserian ganglion. Pain. 1999;79(1):51-58.

22. Scrivani SJ, Keith DA, Mathews ES, Kaban LB. Percutaneous stereotactic differential radiofrequency thermal rhizotomy for the treatment of trigeminal neuralgia. J Oral Maxillofac Surg. 1999;57(2):104-111; discussion 111-112.

23. Kanpolat Y, Savas A, Bekar A, Berk C. Percutaneous controlled radiofrequency trigeminal rhizotomy for the treatment of idiopathic trigeminal neuralgia: 25-year experience with 1,600 patients. Neurosurgery. 2001;48(3):524-534.

24. Fraioli B, Esposito V, Guidetti B, Cruccu G, Manfredi M. Treatment of trigeminal neuralgia by thermocoagulation, glycerolization, and percutaneous compression of the gasserian ganglion and/or retrogasserian rootlets: long-term results and therapeutic protocol. Neurosurgery. 1989;24(2):239-245.

25. Sharma R, Phalak M, Katiyar V, Borkar S, Kale SS, Mahapatra AK. Microvascular decompression versus stereotactic radiosurgery as primary treatment modality for trigeminal neuralgia: A systematic review and meta-analysis of prospective comparative trials. Neurol India. 2018;66(3):688-692; discussion 532-534.

26. Fang L, Ying S, Tao W, Lan M, Xiaotong Y, Nan J. 3D CT-guided pulsed radiofrequency treatment for trigeminal neuralgia. Pain Pract. 2014;14(1):16-21.

27. Luo F, Meng L, Wang T, Yu X, Shen Y, Ji N. Pulsed radiofrequency treatment for idiopathic trigeminal neuralgia: a retrospective analysis of the causes for ineffective pain relief. Eur J Pain. 2013;17(8):1189-1192.

28. Huang J, Ni Z, Finch P. Gasserian ganglion and retrobulbar nerve block in the treatment of ophthalmic postherpetic neuralgia: a case report. Pain Pract. 2017;17(7):961-967.

29. Candido KD, Germanovich A, Ghaly RF, Gorelick GH, Knezevic NN. Case report: computed tomography scan-guided Gasserian ganglion injection of dexamethasone and lidocaine for the treatment of recalcitrant pain associated with herpes simplex type 1 infection of the ophthalmic division of the trigeminal nerve. Anesth Analg. 2011;112(1):224-227.

30. Jafree DJ, Williams AC, Zakrzewska JM. Impact of pain and postoperative complications on patient-reported outcome measures 5 years after microvascular decompression or partial sensory rhizotomy for trigeminal neuralgia. Acta Neurochir (Wien). 2018;160(1):125-134.

31. Elawamy A, Abdalla EEM, Shehata GA. Effects of Pulsed Versus Conventional Versus Combined Radiofrequency for the Treatment of Trigeminal Neuralgia: A Prospective Study. Pain Physician. 2017;20(6):E873-E881
Journal of Pain Research

\section{Publish your work in this journal}

The Journal of Pain Research is an international, peer reviewed, open access, online journal that welcomes laboratory and clinical findings in the fields of pain research and the prevention and management of pain. Original research, reviews, symposium reports, hypothesis formation and commentaries are all considered for publication.
The manuscript management system is completely online and includes a very quick and fair peer-review system, which is all easy to use. Visit http://www.dovepress.com/testimonials.php to read real quotes from published authors. 